doi: $10.15407 /$ ujpe62.09.0779

A.I. LESIUK, M.F. LEDNEY, O.S. TARNAVSKYY

Taras Shevchenko National University of Kyiv

(4, Academician Glushkov Ave., Kyiv 03127, Ukraine; e-mail: lesyuk.andrey@gmail.com,

ledney@univ.kiev.ua,smalko1@bigmir.net)

\title{
ORIENTATIONAL INSTABILITY \\ INDUCED BY THE ELECTRIC FIELD \\ IN A CELL OF A NEMATIC LIQUID CRYSTAL
}

PACS 61.30.Cz, 61.30.Gd,
$6130.306470 \mathrm{mf}$

\begin{abstract}
The orientational instability of the director induced by an external dc electric field in the homeotropic cell including a nematic liquid crystal with negative dielectric anisotropy has been studied. The easy axis gliding in the plane perpendicular to either of polymer cell substrates is taken into consideration. It is explained by the reorientation of the elastic fragments of polymer molecules of the cell substrate owing to the interaction between the permanent or induced dipole moments of the substrate molecules and the electric field. In both cases, the orientational instability of the liquid crystal director is found to have a threshold character. The evolution of the nematic director and the easy axis is analyzed since the moment of electric field switching-on, during the system transition into a stationary state, and until the system relaxes into the initial homogeneous state after the electric field is switched-off. The calculated time dependences of the easy-axis deviation angle are compared with the literature experimental data.

Keywords: nematic liquid crystal, negative dielectric anisotropy, orientational instability, easy axis gliding, switching-on/off time, Fréedericksz transition.
\end{abstract}

\section{Introduction}

Intense researches that were carried out in the domain of liquid crystal (LC) science during the last decade promoted the rapid development of liquid crystal display technologies. Technological applications of LCs are closely connected with a possibility to control the orientational ordering of the mesophase in a $\mathrm{LC}$ cell, which is a basic constructive element of any LC-based information display [1].

Note that the orientational ordering in the LC cell bulk substantially depends on the conditions imposed on the director at the cell surface, in particular, on the direction of the easy orientation axis of the director at the cell substrate. The easy orientation axis of the director, which is induced at the polymer substrate of LC cells, e.g., using the photoalignment method [1,2], was found to be able to change its orientation (to slide) under the influence of an external low-frequency magnetic or electric field. This result extends the pos-

(c) A.I. LESIUK, M.F. LEDNEY, O.S. TARNAVSKYY, 2017

ISSN 2071-0194. Ukr. J. Phys. 2017. Vol. 62, No. 9 sibilities of the easy action on the orientational ordering in the LC cell bulk by creating special conditions for the director at the cell surface, which can be governed by external fields.

Several mechanisms were proposed in order to explain the phenomenon of easy axis gliding. In particular, the easy axis gliding in the plane of the polymer substrate surface (in the azimuthal direction) of a nematic liquid crystal (NLC) cell in the presence of an electric [3] or magnetic [4] field, in the opinion of the cited authors, is a result of the interaction between molecules in the near-surface layer of the nematic and the elastic ("soft") fragments of molecules of a polymer orientant. Owing to this interaction, the influence of the external field on the orientation of the near-surface layer in an NLC brings about a change of the easy axis orientation at the cell substrate.

The same explanation was used to describe the easy axis gliding under the influence of an electric field, but in the direction perpendicular to the surface of the NLC cell substrate (in the polar direction) $[5,6]$. In particular, in work [6], the easy axis gliding on the 
substrate of a cell containing a polymer-stabilized NLC with negative dielectric anisotropy was considered. The cited authors also considered the reorientation dynamics of molecules of a polymer stabilizer, if the latter is deposited onto the cell surface as an orientant, but is not added to the NLC bulk.

Another mechanism driving a change of the easy axis orientation was explained by the adsorption of NLC molecules on the polymer substrate surface. In this case, the influence of an external field on the NLC volume gives rise to the rotation of NLC molecules adsorbed on the surface. As a result of this action, the easy axis becomes shifted by the external electric field at the surface of the nematic cell substrate in both the azimuthal $[7,8]$ and polar [9] directions. The field strengths required for the easy axis to glide and the characteristic times of motions of the director and the easy axis turn out larger by several orders of magnitude, if NLC molecules are adsorbed on the surface of the polymer substrate of the cell in comparison with the case where the adsorption is absent. In particular, in work [9], the characteristic time of the easy axis gliding on the substrate surface associated with the adsorption of NLC molecules was found to have an order of $100 \mathrm{~h}$.

The authors of works [10-12] proposed a phenomenological model for the easy axis reorientation at the surface of a polymer substrate under the influence of an electric field, but in the azimuthal direction. The model is based on the phenomenon of NLC molecule adsorption. It involves the hindering action of a polymer, the viscosity of the easy axis motion, and the influence of the nematic volume on this motion.

The both mechanisms described above were mentioned in work [13], while considering the easy axis gliding in the azimuthal direction of a polymer substrate of the NLC cell under the action of a magnetic field. Besides those factors, the easy axis gliding in the electric field can be stimulated, in particular, by a reorientation of the elastic parts of molecules of the polymer substrate, which takes place due to the interaction of their own or induced dipole moments with the electric field [14]. This model was used in work [15] to explain the creation of an orientational anisotropy on the surface of an electrosensitive polymer by a dc electric field.

In that work, the orientational instability of the director induced by a dc electric field in a cell of a homeotropically aligned NLC with negative dielectric anisotropy was studied. It was assumed that the easy orientation axis of the director at one of the polymer substrates of the cell interacts with the electric field. Aa a result of this interaction, the easy axis glides in the plane oriented perpendicularly to the substrate surface. The contribution of the interaction between the easy axis and the electric field to the surface free energy density of the NLC cell was considered to be a linear or quadratic function of the field strength, depending on whether the dipole moments of the elastic parts of molecules of the polymer substrate are intrinsic or they are induced by the electric field, respectively.

It was found that, irrespective of the interaction between the easy axis and the electric field, the orientational instability of NLC has a threshold behavior. The time dependences for the deviation angles of the director and the moving easy axis were determined since the moment, when the electric field is switched-on, during the period, when the system transits into a stationary state, and until it relaxes into the initial homogeneous state after the field has been switched-off. By comparing the calculated time dependence of the moving easy axis angle and the experimental one [6], the values of such parameters of the system as the energy of the NLC anchoring with a substrate, the viscosity coefficient for the easy axis, and the parameter of the interaction between the easy axis and the electric field were determined.

\section{NLC Free Energy and Equations for the Director Evolution}

Let we have a plane-parallel NLC cell between the planes $z=0$ and $z=L$ with a uniform initial orientation of the director along the axis $O z$. A constant potential difference $U$ is maintained between the cell substrates. The NLC in the cell is characterized by a negative value of anisotropy of its static dielectric permittivity $\left(\epsilon_{a}<0\right)$. The NLC anchoring on the lower $(z=0)$ substrate is uniform and infinitely strong. At the upper $(z=L)$ polymer substrate of the cell, the easy director orientation axis e interacts with the electric field E. As a result of this interaction, the easy axis e glides in the plane oriented perpendicularly to the cell substrate [6].

The free energy of the NLC cell can be written in the form

$F=F_{\mathrm{el}}+F_{\mathrm{E}}+F_{\mathrm{S}}+F_{\mathrm{SE}}$, 
where

$F_{\mathrm{el}}=\frac{1}{2} \int_{V}\left\{K_{1}(\operatorname{div} \mathbf{n})^{2}+K_{2}(\mathbf{n} \operatorname{rot} \mathbf{n})^{2}+\right.$

$\left.+K_{3}[\mathbf{n} \times \operatorname{rot} \mathbf{n}]^{2}\right\} d V$

is the elastic NLC energy;

$F_{\mathrm{E}}=-\frac{1}{8 \pi} \int_{V} \mathbf{E D} d V$

the anisotropic contribution to the free energy from the dc electric field;

$F_{\mathrm{S}}=-\frac{W}{2} \int_{\mathrm{S}}(\mathbf{e n})^{2} d S$

the contribution of the NLC interaction with the upper $(z=L)$ cell substrate to the surface free energy expressed as the Rapini potential;

$F_{\mathrm{SE}}=-\frac{\alpha}{m} \int_{\mathrm{S}}(\mathbf{e E})^{m} d S$

the contribution of the interaction between the easy orientation axis of the director at the upper $(z=L)$ cell substrate and the electric field; $K_{1}, K_{2}$, and $K_{3}$ are the NLC elastic constants; $\mathbf{n}$ is the director; $\mathbf{D}=$ $=\hat{\epsilon} \mathbf{E}$ the electric induction vector; $\hat{\epsilon}=\epsilon_{\perp} \hat{\mathbf{1}}+\epsilon_{a} \mathbf{n} \otimes \mathbf{n}$ is the tensor of NLC static dielectric permittivity; $\epsilon_{a}=\epsilon_{\|}-\epsilon_{\perp}<0$ is its anisotropy; and $W$ the polar energy of anchoring between the NLC and the upper substrate associated with director deviations in the plane $x O z$. The parameter $m$ is put equal to 1 or 2 , depending on whether the polymer orientant molecules are characterized by intrinsic or induced dipole moments, respectively.

Let us consider planar deformations of the NLC director field [6]. The axis $O x$ of the Cartesian coordinate system is so directed that the director deviations lie in the plane $x O z$. Owing to the system uniformity in the plane $x O y$, the director $\mathbf{n}$ in the cell bulk and the easy axis e at the upper cell substrate can be written in the form

$$
\mathbf{n}=\mathbf{i} \sin \theta(z, t)+\mathbf{k} \cos \theta(z, t),
$$$$
\mathbf{e}=\mathbf{i} \sin \psi(t)+\mathbf{k} \cos \psi(t),
$$

where $\theta(z, t)$ and $\psi(t)$ are the deviation angles of the director and the moving easy axis, respectively, from their initial orientation direction along the axis $O z$, and $\mathbf{i}$ and $\mathbf{k}$ are the unit vectors of the Cartesian coordinate system.

The equation for the director should evidently be considered together with the equations for the electric field in the nematic volume. In accordance with the equation $\operatorname{rot} \mathbf{E}=0$, the vector of electric field strength in the NLC bulk has the form $\mathbf{E}=\left(0,0, E_{z}(z)\right)$. Then, as follows from the equation $\operatorname{div} \mathbf{D}=0$, the component $D_{z}=\epsilon_{z z} E_{z}$ is a constant. Since the potential difference equals $U=$ $=\int_{0}^{L} E_{z} d z$, we obtain

$D_{z}=U\left[\int_{0}^{L}\left(\epsilon_{\perp}+\epsilon_{a} \cos ^{2} \theta\right)^{-1} d z\right]^{-1}$.

The free energy (1) per unit of the cell surface area takes the form

$F=\frac{1}{2} \int_{0}^{L}\left(K_{1} \sin ^{2} \theta+K_{3} \cos ^{2} \theta\right)\left(\frac{d \theta}{d z}\right)^{2} d z-\frac{U D_{z}}{8 \pi}-$
$-\frac{W}{2} \cos ^{2}\left(\theta_{L}-\psi\right)-\frac{\alpha D_{z} \cos \psi}{\epsilon_{\perp}+\epsilon_{a} \cos ^{2} \theta_{L}}$

where the component $D_{z}$ of the electric induction vector is given by expression $(3)$, and $\theta_{L}=\theta(z=L)$ is the director deviation angle at the upper cell substrate.

By minimizing the free energy (4) with respect to the angles $\theta$ and $\psi$, we obtain the equation

$$
\begin{aligned}
& \left(K_{1} \sin ^{2} \theta+K_{3} \cos ^{2} \theta\right) \theta_{z z}^{\prime \prime}-\left(K_{3}-K_{1}\right) \theta_{z}^{\prime 2} \sin \theta \cos \theta+ \\
& +\frac{D_{z}^{2}}{U}\left(\frac{U}{4 \pi}+\frac{\alpha \cos \psi}{\epsilon_{\perp}+\epsilon_{a} \cos ^{2} \theta_{L}}\right) \frac{\left|\epsilon_{a}\right| \sin \theta \cos \theta}{\left(\epsilon_{\perp}+\epsilon_{a} \cos ^{2} \theta\right)^{2}}=\eta_{1} \theta_{t}^{\prime}
\end{aligned}
$$

and the corresponding boundary conditions

$\left.\theta\right|_{z=0}=0$,

$\left.\left(K_{1} \sin ^{2} \theta_{L}+K_{3} \cos ^{2} \theta_{L}\right) \theta_{z}^{\prime}\right|_{z=L}+\frac{W}{2} \sin 2\left(\theta_{L}-\psi\right)+$

$+2 \alpha D_{z} \cos \psi \frac{\left|\epsilon_{a}\right| \sin \theta_{L} \cos \theta_{L}}{\left(\epsilon_{\perp}+\epsilon_{a} \cos ^{2} \theta_{L}\right)^{2}}=0$,

$\frac{W}{2} \sin 2\left(\theta_{L}-\psi\right)-\frac{\alpha D_{z} \sin \psi}{\epsilon_{\perp}+\epsilon_{a} \cos ^{2} \theta_{L}}=\eta_{2} \psi_{t}^{\prime}$.

Here, $\eta_{1}$ is the coefficient of NLC bulk viscosity, $\eta_{2}$ the viscosity coefficient for the easy axis, and primes 
on the functions $\theta$ and $\psi$ mean their derivatives with respect to the corresponding arguments. The term on the right-hand side of Eq. (5) describes, as was done in works $[1,16,17]$, dissipative processes occurring in the NLC bulk, when the director rotates, provided that its connection with hydrodynamic motions of the nematic are neglected. Similarly to work [11], the easy axis gliding in the polar direction on the upper $(z=L)$ cell substrate is described by a term on the right-hand side of the boundary condition (8). In the general case, the solution of Eq. (5), which should satisfy the boundary conditions (6)-(8), can be obtained only numerically.

\section{Evolution of the Director and the Moving Easy Axis}

\subsection{Linear interaction between the easy axis and the electric field}

Let the contribution of the interaction between the moving axis e of easy director orientation and the electric field $\mathbf{E}$ at the upper $(z=L)$ cell substrate to the surface free energy of an NLC cell be linear in the electric field strength, i.e. the parameter $m=1$ in expression (1) for $F_{\mathrm{SE}}$. We assume the angles $\theta$ and $\psi$ to be small. Then the behavior of the system is described by Eq. (5) linearized in $\theta$ and $\psi$ :

$\theta_{\xi \xi}^{\prime \prime}+u(u+\tilde{\alpha}) \theta=\theta_{\tau}^{\prime}$

and the linearized boundary conditions (6)-(8)

$\left.\theta\right|_{\xi=0}=0$,

$\left.\theta_{\xi}^{\prime}\right|_{\xi=1}+\varepsilon\left(\theta_{L}-\psi\right)+2 \tilde{\alpha} u \theta_{L}=0$,

$\varepsilon\left(\theta_{L}-\psi\right)-\tilde{\alpha} \nu u \psi=\gamma \psi_{\tau}^{\prime}$.

Here, the following dimensionless parameters are introduced: the coordinate $\xi=z / L$, the time $\tau=$ $=t K_{3} /\left(\eta_{1} L^{2}\right)$, the viscosity coefficient $\gamma=\eta_{2} /\left(\eta_{1} L\right)$, the anchoring energy $\varepsilon=W L / K_{3}$, the potential difference $u=\pi U / U_{\mathrm{th}}^{\infty}$, the interaction parameter $\tilde{\alpha}=\left(\alpha / \epsilon_{\|}\right) \sqrt{4 \pi\left|\epsilon_{a}\right| / K_{3}}$, and $\nu=\epsilon_{\|} /\left|\epsilon_{a}\right|$, where $U_{\mathrm{th}}^{\infty}=\pi \sqrt{4 \pi K_{3} /\left|\epsilon_{a}\right|}$ is the Fréedericksz transition threshold at $W=\infty$ in the absence of easy axis gliding.

The solution of Eq. (9) that satisfies the boundary conditions (10)-(12) describes the deviation angles for the director,

$\theta(\xi, \tau)=\sum_{n=1}^{\infty} A_{n} e^{\Gamma_{n} \tau} \sin \lambda_{n} \xi$ and the moving easy axis,

$$
\psi(\tau)=\sum_{n=1}^{\infty} A_{n}\left(\frac{\lambda_{n}}{\varepsilon} \cos \lambda_{n}+\left(\frac{2}{\varepsilon} \tilde{\alpha} u+1\right) \sin \lambda_{n}\right) e^{\Gamma_{n} \tau},
$$

where $A_{n}$ are integration constants, $\Gamma_{n}=u(u+\tilde{\alpha})-$ $-\lambda_{n}^{2}$, and $\lambda_{n}$ are the positive roots of the equation

$\varepsilon^{2}=\left[\gamma\left(u(u+\tilde{\alpha})-\lambda^{2}\right)+\varepsilon+\nu \tilde{\alpha} u\right](\lambda \operatorname{ctg} \lambda+\varepsilon+2 \tilde{\alpha} u)$.

Note that expression (14) for a deviation angle $\psi(\tau)$ of the easy axis is obtained from the equation

$\gamma \psi_{\tau}^{\prime}+\nu \tilde{\alpha} u \psi=-\left.\theta_{\xi}^{\prime}\right|_{\xi=1}-2 \tilde{\alpha} u \theta$,

which follows from the boundary conditions (11) and (12).

As one can see from Eqs. (13) and (14), if at least one of the $\Gamma$-values is positive $\left(\Gamma_{n}>0\right)$, spatial perturbations of the director field of the type $\sin \lambda_{n} \xi$ will exponentially increase in the system. Therefore, the orientational instability of NLC in the electric field has a threshold character. The orientational instability threshold for the director is determined from the condition $\Gamma_{1}=0$, in which $\lambda_{1}$ is the smallest (by absolute value) root of Eq. (15). From whence, the threshold value is given by the smallest positive root of the equation

$\sqrt{u^{2}+\tilde{\alpha} u} \operatorname{ctg} \sqrt{u^{2}+\tilde{\alpha} u}+\tilde{\alpha} u\left(2+\frac{\nu \varepsilon}{\varepsilon+\tilde{\alpha} \nu u}\right)=0$.

Figure 1 demonstrates the dependences of the threshold voltage $U_{\text {th }}$ of the Fréedericksz transition on the interaction parameter $\alpha$ value that were calculated from Eq. (16) for several values of anchoring energy $\varepsilon$. The threshold magnitude $U_{\text {th }}$ grows with the anchoring energy $\varepsilon$. In the limiting case $\alpha \rightarrow 0$, irrespective of the anchoring energy $\varepsilon$, the threshold voltage $U_{\text {th }}$ approaches a value of $0.5 U_{\mathrm{th}}^{\infty}$, which corresponds to the Fréedericksz transition threshold in the absence of NLC anchoring on the upper $(\varepsilon=0)$ cell substrate, i.e. at $\varepsilon=0$. As the value of the parameter $\alpha$ grows, the magnitude of $U_{\mathrm{th}}$ first increases from $0.5 U_{\text {th }}^{\infty}$ at $\alpha=0$ to a certain maximum and afterward monotonically decreases. In the limiting case of infinitely large $\alpha$, the threshold magnitude $U_{\text {th }}$ asymptotically tends to zero. The maximum value of 
$U_{\text {th }}$ corresponds to the interaction parameter value $\alpha \sim \epsilon_{\|} \sqrt{K_{3} /\left(4 \pi\left|\epsilon_{a}\right|\right)}$. If $\varepsilon$ grows, the positions of the maximum in the dependence $U_{\mathrm{th}}(\alpha)$ shifts toward smaller $\alpha$. The non-monotonic dependence of the threshold voltage $U_{\text {th }}$ on the parameter $\alpha$ is explained by the opposite actions of the electric field on the NLC director and the moving easy axis. Namely, the electric field stabilizes the initial orientation of the moving easy axis, but destabilizes the initial orientation of the director.

If the voltage $U$ slightly exceeds the threshold value $U_{\text {th }}$, the angles $\theta$ and $\psi$ can be considered small. Then their values will be determined by the first $(n=$ 1) harmonic in expressions (13) and (14), respectively. Higher-order harmonics are neglected because of their smallness. From whence, we obtain the characteristic time of the switching-on of the system as

$t_{\mathrm{on}}=\frac{\eta_{1} L^{2} u_{\mathrm{th}}}{K_{3}\left[\left(u_{\mathrm{th}}+\tilde{\alpha}\right) \sigma_{1}-\tilde{\alpha} / 2\right]\left(u^{2}-u_{\mathrm{th}}^{2}\right)}$,

where

$\sigma_{1}=1-\left[2 \rho_{1}^{2}+\nu+\gamma\left(1+2 u_{\mathrm{th}} / \tilde{\alpha}\right)\right] / s_{1}$,

$s_{1}=\left(\rho_{1}^{2}+2 \gamma\right)\left(1+u_{\mathrm{th}} / \tilde{\alpha}\right)+$

$+\left(\nu+2 \rho_{1}\right)\left(\tilde{\alpha} \nu u_{\mathrm{th}}+\left(1+2 \tilde{\alpha} u_{\mathrm{th}}\right) \rho_{1}\right)$,

$u_{\mathrm{th}}=\pi U_{\mathrm{th}} / U_{\mathrm{th}}^{\infty}, \quad \rho_{1}=1+\tilde{\alpha} \nu u_{\mathrm{th}} / \varepsilon$.

The growth of the anchoring energy $\varepsilon$ and the reduction of the interaction parameter $\alpha$ give rise to the increase of the characteristic switching-on time $t_{\text {on }}$, which is also explained by the opposite actions of the electric field on the orientation of the moving easy axis and the NLC director. With an increase of the viscosity coefficients of the NLC bulk and the easy axis, the switching-on time grows. However, this parameter turns out more sensitive to a change of the viscosity coefficient of the easy axis than that of the NLC bulk.

The evolution of the director and the moving easy axis since the voltage switching-on and until the system transits into the stationary state is governed by the applied voltage and the NLC cell parameters. In Fig. 2, the experimental values of the easy axis deviation angle $\psi$ taken from work [6] are depicted. They were measured at some moments, when the system transited into a stationary state under the action of the electric field created by the applied voltage $U=5 \mathrm{~V}=2.4 U_{\mathrm{th}}^{\infty}$. In Fig. 2, the dependence $\psi(t)$ obtained by numerically solving Eq. (5)

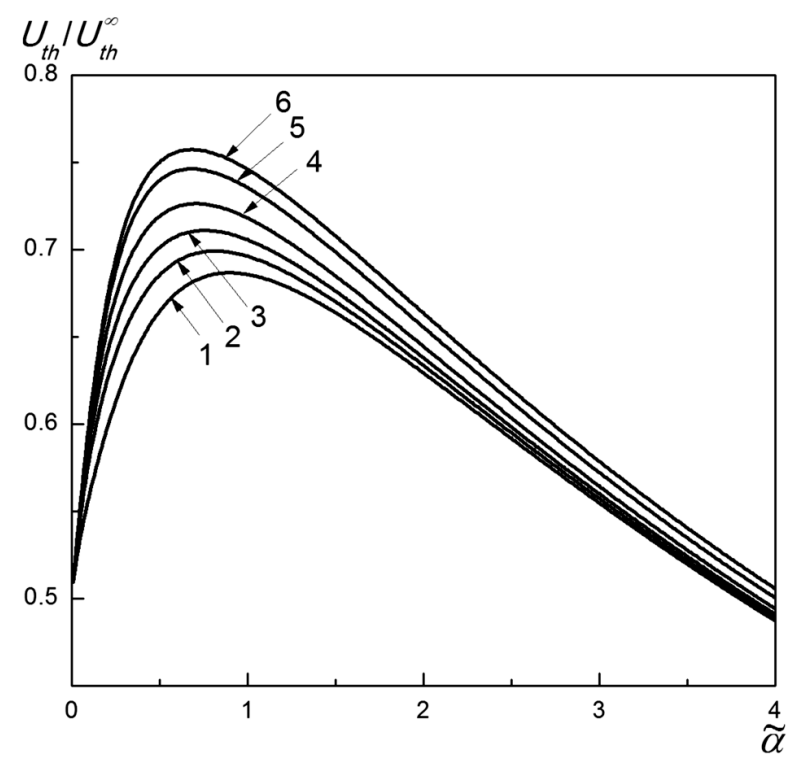

Fig. 1. Dependences of the Fréedericksz transition threshold $U_{\text {th }}$ on the interaction parameter $\alpha$ for $\varepsilon=0.1$ (1), 0.5 (2), 1 (3), 2 (4), 5 (5), and $10(6)$

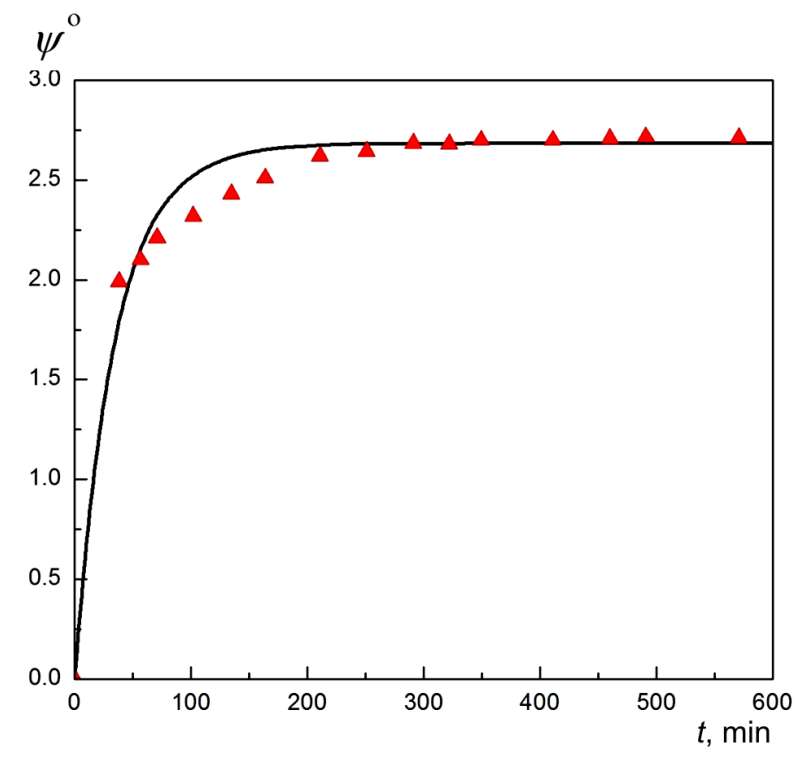

Fig. 2. Comparison of the calculated (solid curve) and experimental (symbols) [6] time dependences of the moving easy axis deviation angle $\psi$ in the case of linear interaction between the easy axis and the electric field at $U=5 \mathrm{~V}$

with the boundary conditions (6)-(8) is also shown for comparison. The calculated dependence $\psi(t)$ was obtained for the following parameter values: the dimensionless anchoring energy $\varepsilon=10$, the dimension- 


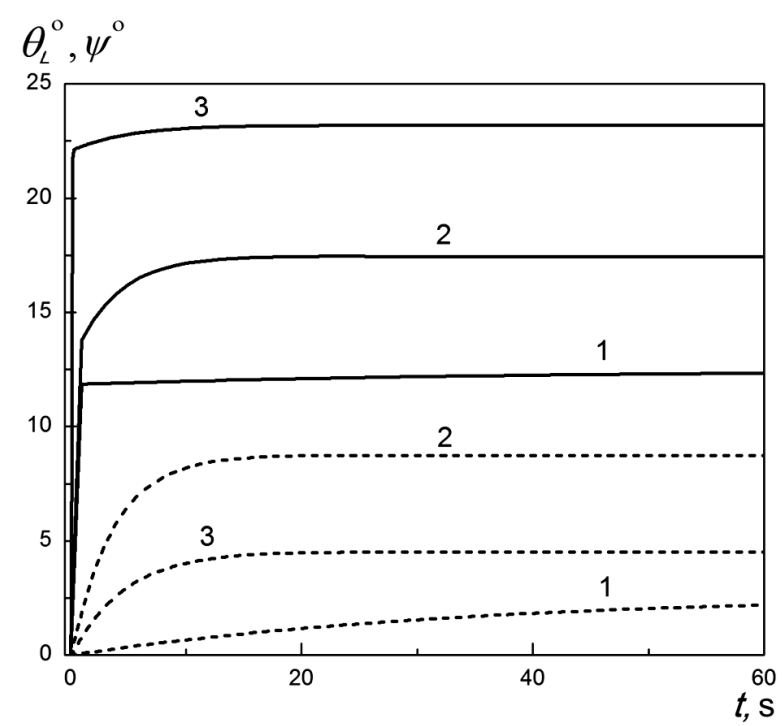

Fig. 3. Time dependences of the moving easy axis, $\psi$, and director, $\theta_{L}$, deviation angles at the cell surface $z=L$ in the electric field with the voltage $U=5 \mathrm{~V}$. The other parameters are: (1) $\varepsilon=10, \tilde{\alpha}=1.77, \eta_{1}=1 \mathrm{P}, \eta_{2}=6 \mathrm{P} \cdot \mathrm{cm}$; (2) $\varepsilon=20$, $\tilde{\alpha}=1, \eta_{1}=1 \mathrm{P}, \eta_{2}=0.4 \mathrm{P} \cdot \mathrm{cm} ;$ (3) $\varepsilon=5, \tilde{\alpha}=1, \eta_{1}=1 \mathrm{P}$, $\eta_{2}=0.4 \mathrm{P} \cdot \mathrm{cm}$

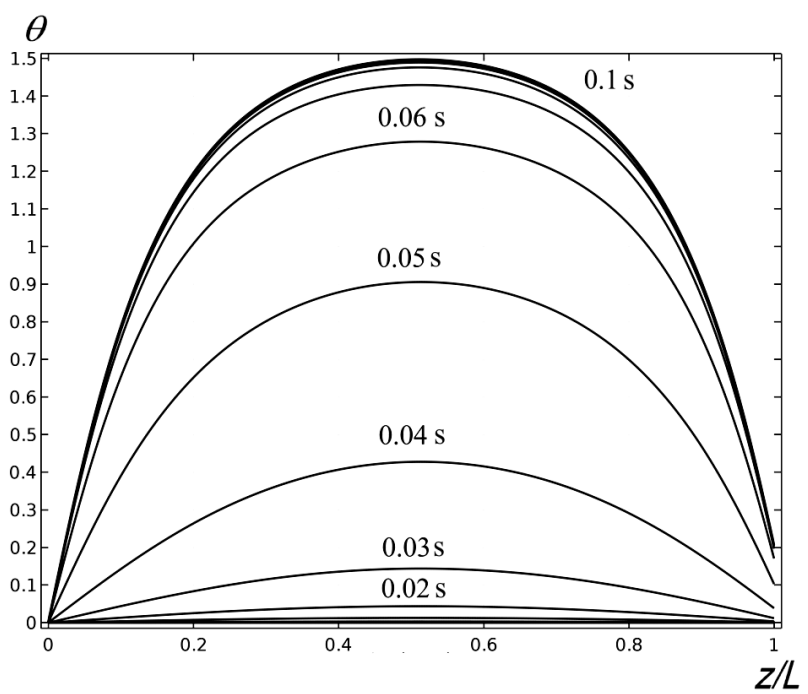

Fig. 4. Evolution of the director deviation angle profile across the cell thickness since the moment of voltage $(U=5 \mathrm{~V})$ applications $(t=0 \mathrm{~s})$ until the transition into the stationary state $(t=0.1 \mathrm{~s}) . \varepsilon=10, \tilde{\alpha}=1.77, \eta_{1}=1 \mathrm{P}, \eta_{2}=6 \mathrm{P} \cdot \mathrm{cm}$

less interaction parameter $\tilde{\alpha}=1.77$, the coefficient of NLC bulk viscosity $\eta_{1}=1 \mathrm{P}$ [18], and the viscosity coefficient of the easy axis $\eta_{2}=160 \mathrm{P} \cdot \mathrm{cm}$. Those values were selected to provide the best agreement between the experimental and theoretical values. In general, the difference between the calculated and experimentally measured values for the angle $\psi$ did not exceed $8 \%$. Furthermore, the obtained anchoring energy $\varepsilon$ agrees with the data of the experimental work [19].

In Fig. 3, the time dependences of deviation angles of the easy axis, $\psi(t)$, and the director, $\theta_{L}(t)$, at the cell surface $z=L$ are exhibited. They were obtained by numerically solving Eq. (5) with the boundary conditions (6)-(8) for various values of NLC cell parameters. Curves 1 correspond to the parameter values calculated above, by using the experimental data in [6]. It was found that the NLC director deviates more eagerly than the easy axis, when the system transits into a stationary state. The moving easy axis traces the director motion, so that $\psi \leqslant \theta_{L}$ (see Fig. 3). It is evident that the easy axis motion is slowed down as a result of its initial orientation stabilization by the electric field.

The director deviation angles across the cell thickness calculated at various moments of the transition into a stationary state are depicted in Fig. 4. Like the case where the easy axis is fixed on both cell substrates, the maximum deformations of the director field are reached in the NLC bulk. As the system approaches the stationary state, the maximum of the director deviation practically does not change its position in the NLC volume. Note that an increase of the easy axis motion viscosity hinders the transition of the system into the stationary state.

In the stationary state $\left(\theta_{t}^{\prime}=\psi_{t}^{\prime}=0\right)$, according to the boundary condition (8), $\theta_{L} \geqslant \psi$ at the upper cell surface, irrespective of the applied potential difference $U$. As follows from the boundary condition (7), $\left.\theta_{z}^{\prime}\right|_{z=L}<0$. Therefore, the maximum of a director deviation is reached in the NLC bulk. Multiplying Eq. (5) by $2 \theta_{z}^{\prime}$ and integrating the result over $z$, we obtain

$\theta_{z}^{\prime}= \pm F\left(\theta, \theta_{m}\right) Q\left(\theta_{m}, \theta_{L}, \psi\right)$,

where

$F\left(\theta, \theta_{m}\right)=\frac{\left(\sin ^{2} \theta_{m}-\sin ^{2} \theta\right)^{1 / 2}}{\left(\epsilon_{\|}-\epsilon_{a} \sin ^{2} \theta\right)^{1 / 2}\left(K_{1} \sin ^{2} \theta+K_{3} \cos ^{2} \theta\right)^{1 / 2}}$,

$Q\left(\theta_{m}, \theta_{L}, \psi\right)=\frac{D_{z}}{\left(\epsilon_{\|}-\epsilon_{a} \sin ^{2} \theta_{m}\right)^{1 / 2}} \times$

$\times\left[\frac{\left|\epsilon_{a}\right|}{4 \pi K_{3}}\left(1+\frac{4 \pi \alpha}{U} \frac{\cos \psi}{\epsilon_{\|}-\epsilon_{a} \sin ^{2} \theta_{L}}\right)\right]^{1 / 2}$,

ISSN 2071-0194. Ukr. J. Phys. 2017. Vol. 62, No. 9 
and $\theta_{m}$ is the maximum of the director deviation angle.

Separating the variables in Eq. (18) and integrating the result over $z$ from 0 to $L$, we obtain

$2 \int_{0}^{\theta_{m}} F\left(\theta, \theta_{m}\right) d z-\int_{0}^{\theta_{L}} F\left(\theta, \theta_{m}\right) d z=L Q\left(\theta_{m}, \theta_{L}, \psi\right)$.

By considering Eq. (19) together with the boundary conditions (7) and (8), which accounts for the value of the derivative $\theta_{z}^{\prime}(18)$ at the cell surface $z=L$, we find the angles $\theta_{m}, \theta_{L}$, and $\psi$. With those values from Eq. (18), we obtain the profile of the director deviation angle $\theta$ across the cell thickness for the given voltage $U$.

The calculated dependences $\theta(z)$ for various values of the applied voltage $U$ and the interaction parameter $\alpha$ are shown in Fig. 5. Increase of the anchoring energy $\varepsilon$ and in the applied voltage $U$ results in a growth of deformations of the NLC director field. When the voltage $U$ grows, the deviation angles of the easy axis, $\psi$, and the director, $\theta_{L}$, at the surface $z=L$ monotonically increase, but $\theta_{L} \geqslant \psi$ everytime. If the interaction parameter $\alpha$ grows at a fixed voltage, the deviation angle $\theta_{L}$ of the director at the surface $z=L$ decreases, which gives rises to larger deformations of the director field in the cell volume.

Now, let us consider the relaxation of the system from the stationary state into the initial uniform one, when the voltage is switched-off. First, let us consider the case where the applied voltage $U$ slightly exceeds its threshold value $U_{\mathrm{th}}$. Then the process of relaxation can be described in the approximation of small $\theta$ and $\psi$ angles, by using the linearized Eq. (9) with the boundary conditions (10)-(12), in which $U=0$. The required, finite at $\tau \rightarrow+\infty$, solution for the deviation angles of the director and the moving easy axis looks like

$$
\begin{aligned}
& \theta(\xi, \tau)=\sum_{n=1}^{\infty} A_{n} e^{-\varkappa_{n}^{2} \tau} \sin \varkappa_{n} \xi, \\
& \psi(\tau)=\sum_{n=1}^{\infty} \frac{A_{n}}{\gamma \varkappa_{n}} e^{-\varkappa_{n}^{2} \tau} \cos \varkappa_{n},
\end{aligned}
$$

where $A_{n}$ are integration constants, and $\kappa_{n}$ are positive roots of the equation

$\tan \varkappa=\frac{1}{\gamma \varkappa}-\frac{\varkappa}{\varepsilon}$. $\theta$

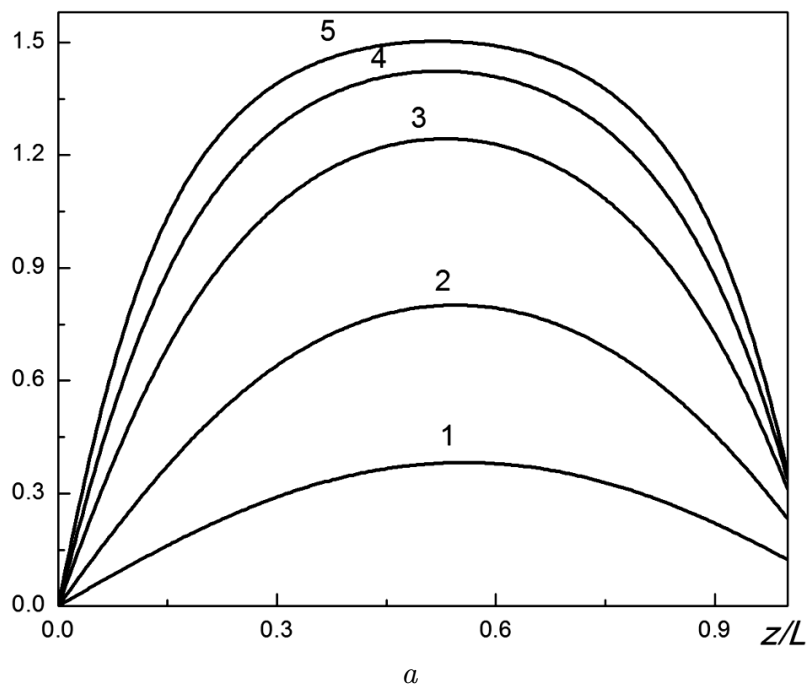

$\theta$

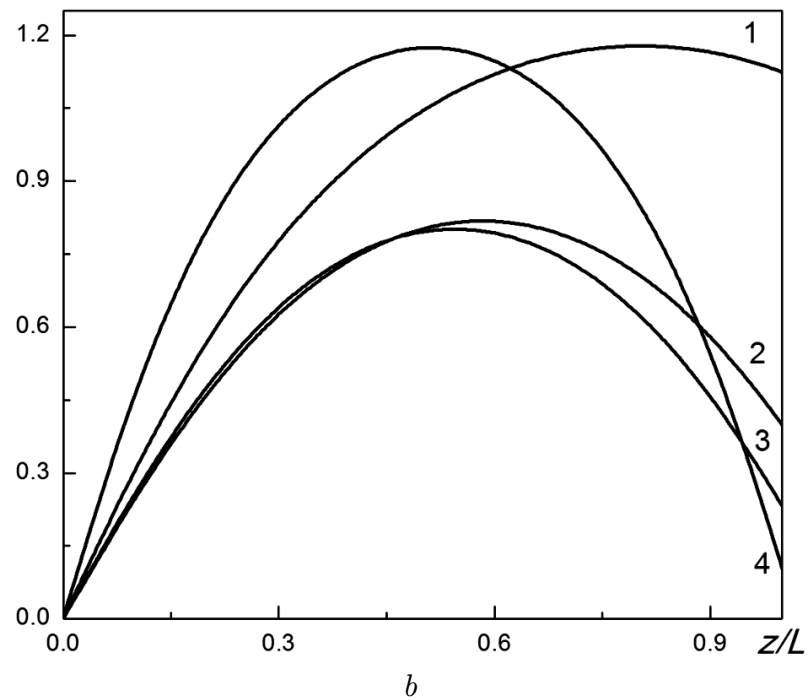

Fig. 5. Dependences of the director deviation angle on the coordinate $z$ at $\varepsilon=10$ and $(a) \tilde{\alpha}=1.77 ; U / U_{\mathrm{th}}^{\infty}=0.8(1), 1$ (2), 1.5 (3), 2(4), 2.5(5); (b) $U / U_{\mathrm{th}}^{\infty}=1 ; \tilde{\alpha}=0.1(1), 0.5$ (2), $1(3), 5(4)$

As follows from Eq. (20), the characteristic time of relaxation is determined by the most slowly damped mode (with $n=1$ ), and it is equal to

$t_{\mathrm{off}}=\frac{\eta_{1} L^{2}}{\varkappa_{1}^{2} K_{3}}$.

Although the relaxation time $t_{\text {off }}$ is practically independent of the interaction parameter $\alpha$, the possi- 


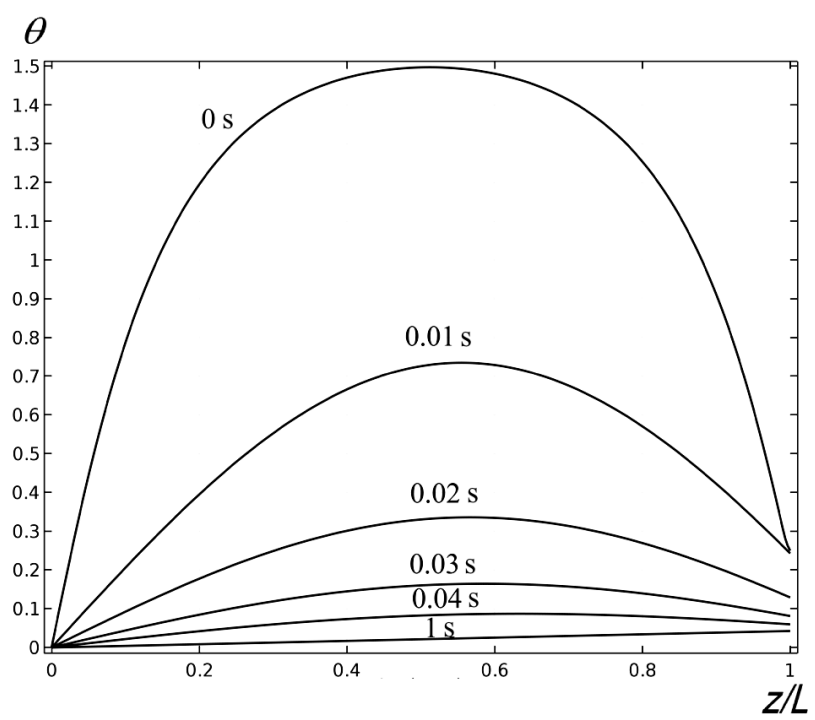

Fig. 6. Evolution of the director deviation angle profile across the cell thickness during the first second after the voltage switching-off. The total relaxation time of the system has an order of $1000 \mathrm{~s} ; \varepsilon=10, \tilde{\alpha}=1.77, \eta_{1}=1 \mathrm{P}, \eta_{2}=6 \mathrm{P} \cdot \mathrm{cm}$

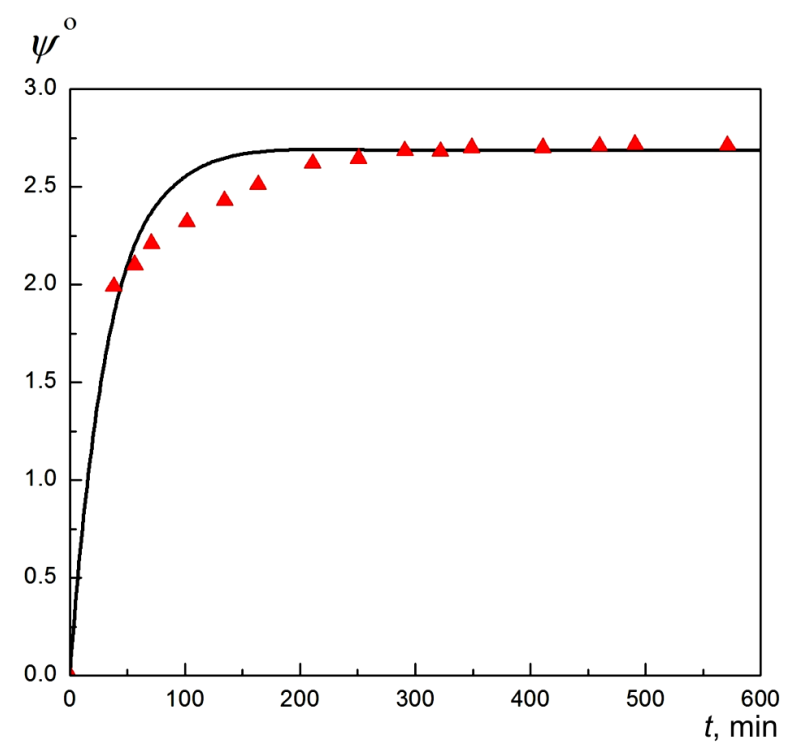

Fig. 7. Comparison of the calculated (solid curve) and experimental (symbols) [6] time dependences of the moving easy axis deviation angle $\psi$ in the case of quadratic interaction between the easy axis and the electric field at $U=5 \mathrm{~V}$

bility of the easy axis gliding gives rise to its growth in comparison with the case without the gliding. In particular, in the latter case at $W=\infty$, the relaxation time equals $t_{\text {off }}^{\infty}=\eta_{1} L^{2} /\left(\pi^{2} K_{3}\right)<t_{\text {off }}$, so that $t_{\text {off }}^{\infty}<t_{\text {off }}$, since $\varkappa_{1}<\pi$. An increase of the anchoring energy $\varepsilon$ results in a reduction of the relaxation time $t_{\text {off }}$, which is explained by an enhancement of the interaction between the director and the easy axis.

Figure 6 exhibits the director deviation angles across the cell thickness, which were calculated for various time moments after the voltage in the system that was in a stationary state was switched-off. As one can see, the director deviation maximum moves rather quickly from the NLC bulk to the substrate with the moving easy axis. In particular, the dependence of the director deviation angle $\theta(z, t)$ becomes almost linear in $z$ within rather a short time interval (about $1 \mathrm{~s}$ ) in comparison with the time of the total relaxation of the system into the initial uniform state (of an order of $1000 \mathrm{~s}$ ). The latter value is evidently associated with a relatively large value of the easy axis motion viscosity coefficient and is close to the value obtained in work [11]. In general, as the system relaxes, the director returns more quickly to the initial uniform state and drags the easy axis behind it, so that $\theta_{L} \leqslant \psi$.

\subsection{Quadratic interaction between the easy axis and the electric field}

Let $\mathrm{c}$ contribution of the interaction between the axis of easy director orientation and the electric field at the polymer substrate $z=L$ to the surface free energy of an NLC cell be quadratic in the electric field strength $E$, i.e. the parameter $m=2$ in expression (1). By minimizing the free energy (1) with respect to the angles $\theta$ and $\psi$, we arrive at the equation

$$
\begin{aligned}
& \left(K_{1} \sin ^{2} \theta+K_{3} \cos ^{2} \theta\right) \theta_{z z}^{\prime \prime}-\left(K_{3}-K_{1}\right) \theta_{z}^{\prime 2} \sin \theta \cos \theta+ \\
& +\frac{D_{z}^{2}}{U}\left(\frac{U}{4 \pi}+\frac{2 \alpha D_{z} \cos ^{2} \psi}{\left(\epsilon_{\perp}+\epsilon_{a} \cos ^{2} \theta_{L}\right)^{2}}\right) \times \\
& \times \frac{\left|\epsilon_{a}\right| \sin \theta \cos \theta}{\left(\epsilon_{\perp}+\epsilon_{a} \cos ^{2} \theta\right)^{2}}=\eta_{1} \theta_{t}^{\prime}
\end{aligned}
$$

with the boundary conditions

$\left.\theta\right|_{z=0}=0$,

$$
\begin{aligned}
& \left.\left(K_{1} \sin ^{2} \theta_{L}+K_{3} \cos ^{2} \theta_{L}\right) \theta_{z}^{\prime}\right|_{z=L}+\frac{W}{2} \sin 2\left(\theta_{L}-\psi\right)+ \\
& +2 \alpha D_{z}^{2} \cos ^{2} \psi \frac{\left|\epsilon_{a}\right| \sin \theta_{L} \cos \theta_{L}}{\left(\epsilon_{\perp}+\epsilon_{a} \cos ^{2} \theta_{L}\right)^{3}}=0 \\
& \frac{W}{2} \sin 2\left(\theta_{L}-\psi\right)-\alpha D_{z}^{2} \frac{\sin \psi \cos \psi}{\left(\epsilon_{\perp}+\epsilon_{a} \cos ^{2} \theta_{L}\right)^{2}}=\eta_{2} \psi_{t}^{\prime},
\end{aligned}
$$


As was in the linear case considered above, the orientational instability of NLC in the electric field has a threshold. From the linearized equation (23) and the boundary conditions (24)-(26), it follows that the magnitude of the Fréedericksz transition threshold is equal to the smallest positive root of the equation

$u \sqrt{1+2 \tilde{\alpha}} \operatorname{ctg}(u \sqrt{1+2 \tilde{\alpha}})+\tilde{\alpha} u^{2}\left(2+\frac{\nu \varepsilon}{\varepsilon+\tilde{\alpha} \nu u^{2}}\right)=0$

where $\tilde{\alpha}=4 \pi \alpha /\left(\epsilon_{\|} L\right)$. The dependence of the threshold voltage $U_{\text {th }}$ for the Fréedericksz transition on the interaction parameter $\alpha$ determined from Eq. (27) turns out qualitatively similar to that depicted in Fig. 1 for the case of linear interaction between the moving easy axis and the electric field. In particular, in the absence of this interaction $(\alpha=0)$, the threshold voltage amounts to $0.5 U_{\text {th }}^{\infty}$. Larger anchoring energy values lead to a growth of the orientational instability threshold.

If the voltage $U$ slightly exceeds its threshold value $U_{\text {th }}$, then, assuming the deviation angles of the director, $\theta$, and the easy orientation axis, $\psi$, to be small and considering the linearized problem, we obtain the following characteristic time of the switching-on:

$t_{\mathrm{on}}=\frac{\eta_{1} L^{2}}{K_{3} \sigma_{2}(1+2 \tilde{\alpha})\left(u^{2}-u_{\mathrm{th}}^{2}\right)}$,

where

$\sigma_{2}=1-2\left[2 \rho_{2}^{2}+\nu+\gamma(2+1 / \tilde{\alpha})\right] / s_{2}$,

$s_{2}=\left(\rho_{2}^{2}+2 \gamma\right)(2+1 / \tilde{\alpha})+$

$+\left(\nu+2 \rho_{2}\right)\left(\tilde{\alpha} \nu u_{\text {th }}^{2}+\left(1+2 \tilde{\alpha} u_{\text {th }}^{2}\right) \rho_{2}\right)$,

$\rho_{2}=1+\tilde{\alpha} \nu u_{\mathrm{th}}^{2} / \varepsilon$.

The dependence of the switching-on time $t_{\text {on }}$ on the NLC cell parameters turns out qualitatively similar to the case of linear interaction between the easy axis and the electric field. For instance, the time $t_{\text {on }}$ grows with the anchoring energy $\varepsilon$ and the coefficients of NLC bulk, $\eta_{1}$, and easy axis, $\eta_{2}$, motion viscosity, as well as with a decrease of the interaction parameter $\alpha$. The variation of the coefficient of NLC bulk vis$\operatorname{cosity} \eta_{1}$ within its admitted region weakly affects the $t_{\text {on }}$ magnitude.

In Fig. 7, a comparison is made between the values of the deviation angle $\psi$ obtained experimentally [6] and determined from Eq. (23) for the time interval since the voltage in the system has been switchedon and until the system transits into its stationary state. The calculated dependence $\psi(t)$ was obtained for the following parameter values: the dimensionless anchoring energy $\varepsilon=10$, the dimensionless interaction parameter $\tilde{\alpha}=0.19$, the coefficient of NLC bulk viscosity $\eta_{1}=1 \mathrm{P}$ [18], and the coefficient of easy axis motion viscosity $\eta_{2}=160 \mathrm{P} \cdot \mathrm{cm}$. The indicated values of NLC cell parameters were selected to provide the best agreement between the experimental and theoretical values of $\psi$. The difference between them did not exceed $7 \%$.

As a whole, the results of calculations testify that the evolution of the director and moving easy axis deviation angles and its dependence on the NLC cell parameters and the applied voltage is qualitatively similar to the case considered above for the linear interaction between the easy axis and the electric field.

\section{Conclusions}

The director reorientation from the homeotropic state in a planar NLC cell with negative dielectric anisotropy under the acton of a dc electric field has been studied. The gliding of the axis of easy director orientation on one of the polymer cell substrates in a plane perpendicular to the substrate is taken into account. The easy axis gliding arises owing to the interaction between the easy axis and the electric field. The contribution of this interaction to the density of the surface free energy of an NLC cell is taken to be linear or quadratic in the electric field strength, in accordance to whether the dipole moments of the elastic parts of polymer substrate molecules are intrinsic or induced by the electric field.

Irrespective of the interaction between the moving easy axis and the electric field, the orientational instability of NLC has a threshold character. The orientational instability threshold decreases with a reduction of the energy of the NLC anchoring on the substrate, on which the easy axis moves. In the case where the easy axis does not interact with the electric field, the Fréedericksz transition threshold tends to its value obtained in the absence of an NLC anchoring on the substrate with the moving easy axis $(\varepsilon=0)$. If the parameter $\alpha$ of the easy axis interaction with the electric field increases from zero, 
the threshold value first increases to a certain maximum value and then decreases to zero at infinitely large $\alpha$. The maximum threshold value is attained at $\alpha \sim \epsilon_{\|} \sqrt{K_{3} /\left(4 \pi\left|\epsilon_{a}\right|\right)}$.

The evolution of both the director and the moving easy axis is governed by the applied voltage $U$, interaction parameter $\alpha$, anchoring energy $\varepsilon$, and the coefficients of NLC bulk and easy axis motion viscosity. The time dependences turn out qualitatively similar in the both cases of easy axis interaction with the electric field. Analytical expressions are derived for the time dependences of the director and easy axis deviation angles after the electric field switching-on/off as solutions of the linearized variational equation and the linearized boundary conditions.

Since the voltage application moment and until the system transits into a stationary state, the director deviates more rapidly and drags the easy axis behind it in the both cases of easy axis interaction with the electric field. The maximum deformations of the director field are reached in the NLC bulk and practically do not change their position, when the system transits into a stationary state. By comparing the time dependences calculated for the easy axis deviation angle within the period, when the system transits into a stationary state with the corresponding experimental ones [6], the values of the anchoring energy $\varepsilon$, the coefficient of easy axis motion viscosity, and the parameter $\alpha$ of the easy axis interaction with the electric field are determined. The characteristic time of switching-on of the system, $t_{\mathrm{on}}$, is found to decrease with an increase of the interaction parameter $\alpha$, as well as with a reduction of the anchoring en$\operatorname{ergy} \varepsilon$ and the coefficients of NLC bulk and easy axis motion viscosity.

In the stationary state, the largest deformations of the director field takes place in the NLC bulk, irrespective of the interaction between the easy axis and the electric field. An increase of the anchoring energy $\varepsilon$ and the interaction parameter $\alpha$ results in larger deformations of the director field.

Irrespective of the interaction of the easy axis with the electric field, if the system is in a stationary state and the voltage is switched-off, an almost linear distribution of the director deviation angle across the cell thickness is established rather quickly in comparison with the time of total relaxation of the system. The director deviation maximum turns out shifted toward the surface with the moving easy axis. In the both cases of interaction between the easy axis and the electric field, the characteristic time of relaxation $t_{\text {off }}$ was found to be identical and independent of the interaction parameter $\alpha$. As the anchoring energy increases and the coefficients of NLC bulk and easy axis motion viscosity decrease, the time $t_{\text {off }}$ diminishes.

The authors express their gratitude to I.P. Pinkevych for useful remarks, when discussing the results of this work.

1. Deng-Ke Yang, Shin-Tson Wu. Fundamentals of Liquid Crystal Devices (Wiley, 2015).

2. O. Yaroshchuk, Yu. Reznikov. Photoalignment of liquid crystals: Basics and current trends. J. Mater. Chem. 22, 286 (2012).

3. Yu. Kurioz, V. Reshetniak, Yu. Reznikov. Orientation of a liquid crystal on a soft photoaligning surface. Mol. Cryst. Liq. Cryst. 375, 535 (2002).

4. I. Janossy, T. I. Kosa. Gliding of liquid crystals on soft polymer surfaces. Phys. Rev. E 70, 052701 (2004).

5. K. Antonova, K. Slyusarenko, O. Buluy, Ch. Blanc, S. Joly, Yu. Reznikov, M. Nobili. Aging in glassy polymer-liquidcrystal layers. Phys. Rev. E 83, 050701R (2011).

6. Ji-H. Lee, T.-H. Yoon. Surface gliding of the easy axis of a polymer-stabilized nematic liquid crystal and its dependence on the constituent monomers. Phys. Rev. E 84, 051701 (2011).

7. P. Vetter, Y. Ohmura, T. Uchida. Study of memory alignment of nematic liquid crystals on polyvinyl alcohol coatings. Jpn. J. Appl. Phys. 32, L1239 (1993).

8. S. Faetti, M. Nobili, I. Raggi. Surface reorientation dynamics of nematic liquid crystals. Eur. Phys. J. B 11, 445 (1999).

9. S. Joly, K. Antonova, Ph. Martinot-Lagarde, I. Dozov. Zenithal gliding of the easy axis of a nematic liquid crystal. Phys. Rev. E 70, 050701R (2004).

10. S.V. Pasechnik, V.G. Chigrinov, D.V. Shmeliova et al. Slow relaxation processes in nematic liquid crystals at weak surface anchoring. Liq. Cryst. 33, 175 (2006).

11. S.V. Pasechnik, A.V. Dubtsov, D.V. Shmeliova et al. Effect of combined action of electric field and light on gliding of the easy axis in nematic liquid crystals. Liq. Cryst. 35, 569 (2008).

12. S.V. Pasechnik, A.V. Dubtsov, D.V. Shmeliova et al. Modeling reorientation dynamics of electrically assisted lightinduced gliding of nematic liquid-crystal easy axis. Adv. Condens. Matter Phys. 2013, 363157 (2013).

13. O. Buluy, Y. Reznikov, K. Slyusarenko et al. Formation and dynamics of easy orientation axis in magnetic field on PVCN-F surface. Opto-Elect. Rev. 14, 293 (2006).

ISSN 2071-0194. Ukr. J. Phys. 2017. Vol. 62, No. 9 
14. N.A. Davidenko, I.I. Davidenko, I.A. Savchenko et al. Electro-optical effect in films of azobenzene polycomplexes with cobalt. J. Appl. Phys. 103, 094323 (2008).

15. D. Kasyanyuk, N. Davidenko, Y. Kurioz et al. Electro- and photosensitive azopolymer for alignment of liquid crystals. Opt. Express 23, 26660 (2015).

16. B.Ya. Zeldovich, N.V. Tabiryan, Yu.S. Chilingaryan. Lightfield-induced Fréedericksz transition. Zh. Ėksp. Teor. Fiz. 81, 72 (1981) (in Russian).

17. B.Ya. Zeldovich, N.V. Tabiryan. Theory of light-induced Fréedericksz transition. Zh. Èksp. Teor. Fiz. 82, 1126 (1982) (in Russian).

18. P.A. Breddels, J.C.H. Mulkens. The determination of the Frank elastic constant for twist deformation of 4'-n-pentyl4-cyanobiphenyl(5CB) using a conoscope. Mol. Cryst. Liq. Cryst. 147, 107 (1987).

19. L. Weng, P.-Ch. Liao, L.-Ch. Chien. Surface anchoring of vertical alignment liquid crystal displays enhanced by surface polymer stabilization. SID 2014 Digest P-131 (2014).

Received 05.06.17. Translated from Ukrainian by O.I. Voitenko
А.І. Лесюк, М.Ф. Ледней, О.С. Тарнавсъкий ОРІЄНТАЦІЙНА НЕСТІЙКІСТЬ В КОМІРЦІ НЕМАТИЧНОГО РІДКОГО КРИСТАЛА 3 ВІД'ЄМНОЮ ДІЕЛЕКТРИЧНОЮ АНІЗОТРОПІЕЮ В ЕЛЕКТРИЧНОМУ ПОЛІ

Р е $з$ ю м е

Досліджується орієнтаційна нестійкість директора в гомеотропній комірці нематичного рідкого кристала з від'ємною діелектричною анізотропією в постійному електричному полі. Враховується проковзування осі легкого орієнтування директора в площині, перпендикулярній до однієї з полімерних підкладок комірки. Проковзування легкої осі зумовлено переорієнтацією еластичних частин молекул полімерної підкладки, що є наслідком взаємодії з електричним полем їх власних або наведених дипольних моментів. Встановлено, що в обох випадках взаємодії орієнтаційна нестійкість директора рідкого кристала є пороговою. Досліджена часова поведінка директора і легкої осі з моменту ввімкнення електричного поля з наступним виходом системи в стаціонарний стан та закінчуючи поверненням системи в вихідний однорідний стан після вимкнення напруги. Проведено порівняння розрахункових часових залежностей кута відхилення легкої осі з відповідними даними експериментальних робіт. 